\title{
Importance and challenges of environmental ligand binding and exchange: Introducing single molecule imaging as a model characterization technique
}

By

${ }^{1}$ Navid B. Saleh, ${ }^{1}$ Dipesh Das, ${ }^{1}$ Jaime Plazas-Tuttle, ${ }^{2}$ Darwin Yang, ${ }^{2}$ Jackson Travis DelBonisO'Donnell, and ${ }^{2,3}$ Markita P. Landry ${ }^{*}$

${ }^{1}$ Department of Civil, Architectural and Environmental Engineering, University of Texas at Austin, Austin, TX 78712

${ }^{2}$ Department of Chemical and Biomolecular Engineering, University of California-Berkeley, Berkeley, CA 94720

${ }^{3}$ California Institute for Quantitative Biosciences (qb3), University of California-Berkeley, Berkeley, CA 94720

*Corresponding author: Markita P. Landry, email: landry@berkeley.edu, phone: (510) 6647627 


\section{Abstract}

Highly surface active nano-scale materials, when released into the natural environment, tend to adsorb geo- and bio-macromolecules and end up presenting a modified interface to biological species. Capped nanocrystals and polymer/surfactant modified nanomaterialsalso are known to undergo ligand exchange when exposed to natural systems. Thus, nano-bio interactionswill primarily be governed by the adsorbed or exchanged natural macromolecules. To-date there has been no established technique determining the kinetics of ligand exchange orcharacterizing the bound geo-biomacromolecular corona in an environmental setting. Single-molecule imaging utilizing near-infra red spectrometry, and single-molecule imaging of fluorophore-tagged polymeric ligands can enable detailed characterization of biopolymeric corona. This perspective aims to highlight the importance of ligand exchange, identify roles of surface ligands on nano-bio interaction, and present initial evidence of macromolecular characterization on nanotube surfaces using single-molecule techniques. This commentary also aims to outline the challenges facing nano-environmental health and safety community on assessing biological interaction with complex nano-scale heterostructures in a realistic environmental matrix. 


\section{Introduction}

Physicochemical transformation of nanomaterials (NMs) is inevitable when released into the natural environment ${ }^{1}$, which strongly influences the interaction of these surface-active materials at the environmental and biological interfaces ${ }^{2}$.Geo- and bio-macromolecules present in natural waters will interact with NM surfaces and will adsorb either via direct binding onto pristine or covalently functionalized materials or by replacementof pre-existing engineered ligandsof capped nanocrystals ${ }^{3-9}$.Thus,biological systems and their membranes will interact with NMs first at the interface of the geo- or bio-molecular corona the NM has accrued prior to reaching the biological structure in question. Detailed characterization of this corona is essential for nano environmental health and safety (EHS) studies in complex but realistic natural environmental conditions.

Reduction in free energy by bringing in a monomer to a surface has been identified to be relatively large compared to its thermal energy, hence is known to drive macromolecular adsorption onto colloidal surfaces ${ }^{10}$. Naturally occurring geo- and bio-macromolecules are subjected to solid-liquid distribution in geochemical systems due totheir amphiphilicity.During adsorption process a net reduction in free energy is achieved by expulsion of water molecules; this is attained via a reduction in enthalpy (through elimination of van der Waals forces between water molecules and hydrophobic components), which is somewhat compromised by entropic loss (via dislodgement of oriented water molecules) ${ }^{1}$.Presence of high surface area NMs when suspended in natural waters ${ }^{11}$ enhances this adsorption process. NOM when at the vicinity of a surface introduces strong van der Waals interaction between the sorptive surface and the sorbing high molecular weight

macromolecule. ${ }^{12}$. Furthermore,hydrogen bonding between the NOM functional groups and 
surface moieties can drive macromolecules toward interfaces ${ }^{13}$.Thus,preferential adsorption of NOM onto uncoated NM surfaces is a likely outcome, which will govern the interfacial interaction of these materials in the environment.

Metal and metal oxide nanocrystals are mostly capped with polymers and polymeric surfactants.Theinteraction of these pre-existing ligands with naturally occurring macromolecules mayundergo via partial or complete ligand exchange ${ }^{1}$.Such exchange will be dictated by ligand and macromolecular types and will be modulated by the surrounding environmental matrix. Energetic gain due to replacement of relatively smaller ligands with larger $\mathrm{NOM}^{13}$ or reaction ${ }^{14,15}$ of humic functional moieties with those at the capping agents will determine the kinetics and extent of ligand exchange. Furthermore, NM physicochemical properties will also influence NOM adsorption and ligand exchange processes ${ }^{16}$, where complexity in emergent nano-heterostructures present an additional degree of complexity ${ }^{16-19}$. These key factors of the ligand exchange process will eventually result in the evolution of NM surface chemistry and thus the eventual nano-bio interaction.

Irrespective of whether the NOM will bind to NM surfaces by adsorption or via ligand exchange, the corona formed on these surfaces necessitate detailed characterization.To-date, indirect approaches have been utilized in characterizing geo- and bio-macromolecules on NM surfaces; e.g., Tracking protein, surfactant, and polymer coatings has been accomplished via attenuated total reflection-FTIR, absorption, and bulk fluorescence techniques, which have evaluated desorption of these ligands from various nanomaterial surfaces ${ }^{20-24}$. Additionally, calorimetry has been used in conjunction with electrophoresis to measure protein adsorption on NM, and small angle neutron scattering experiments to measure nanomaterial-adsorbed polymers $^{25,}{ }^{26}$. Fourier transformedinfrared (FTIR) spectroscopy ${ }^{27}$, interfacial force measurements $^{28}$, ellipsometry ${ }^{29}$, contact stylus instruments ${ }^{30}$, are prominent experimental techniques while theoretical modeling ${ }^{31}$ of bound NOM layers provide further insight into 
these complex corona. However, a robust technique is yet to be developed to directly characterize the conformation of the macromolecules on NM surfaces, kinetics of ligand exchange, and extent of macromolecular adsorption or exchange.Although many tools exist to characterize both NMs and the interfacial synthetic or biological coronas ${ }^{32}$, these methods are currently unable to give a detailed picture of biomolecular structure at the nano-bio interface ${ }^{33}$. As a result, the molecular basis for local electronic properties, bioavailability, toxicological effects, structure and conformation of biomolecules on NMs remain unclear ${ }^{34}$, 35.

Single-molecule imaging, utilizing near-infra red spectrometry and visible fluorophores, can enable detailed characterization of optically dense biopolymeric corona ${ }^{36}$. Single molecule imaging is a powerful technique to study individual molecules and singular intermolecular interactions ${ }^{37,38}$. In particular, single-molecule fluorescence microscopy can be used to study individual polymers and macromoleculesand their interactions both in vitro $^{39,40}$ and in biologically complex environments ${ }^{41-43}$. Single-molecule total internal reflection fluorescence microscopy (smTIRF) can achieve sub-diffraction limited imaging resolution by imaging molecules within a 100 nm-deep field of view that isexcited by an evanescent field, therebyeliminating signal from out-of-focus fluorophores. smTIRF allows nanometer spatial resolution and millisecond temporal resolution of single fluorescentlylabeled polymers. As we show here, we can extend this technique to also spatially and temporally resolve interactions ofpolymers, and ligands, both amongst themselves and with synthetic nano-scale materials ${ }^{44}$.

The focus of this perspective is to introduce single molecule imaging as an effective technique for bio-corona characterization. Differences in NOM chemical composition based on variation in natural water compartments is characterized,and the role of corona composition on environmental and nano-bio interaction is also analyzed. This article also 
highlights the challenges associated with characterization of complex nano heterostructures. A brief description of single molecule imaging with smTIRFand preliminary corona characterization data are presented. Strategies are discussed for adoption and utilization of this technique to study the detailed kinetics, extent and conformation of natural macromolecule adsorption, and corona formation on NM surfaces.

\section{Environmental macromolecules and ligand exchange}

The chemistry of naturally occurring bio- and geo-macromolecules widely vary depending on the phase of origin, i.e., terrestrial vs. freshwater or marine aquatic environment, and other environmental parameters ${ }^{45}$. These natural macromolecules can be broadly classified as nonhumified (that originate from minor alteration via decay of tissue from living organisms) and humified (that are decomposition products of non-humified constituents) substances ${ }^{46}$. Carbohydrates, amino acids, proteins, lignin, hormones and low molecular organic acids are the first degradation products or non-humified substances that decomposes further to humified humic acids, fulvic acids, and humins (the combination is known as NOM).Among these natural macromolecules, NOM and polysaccharides are the most ubiquitous, which also are composed of a wide variety of functional groups and can demonstrate anionic/cationic as well as hydrophilic/hydrophobic behavior,depending on their chemical structure ${ }^{47,}{ }^{48}$. Thus, appreciation of the complex and variable chemistry of the geo- and bio-macromolecules when studying NM interaction is critical.

In a natural waterbody, the organic components can either be dissolved or suspended as particulates. The non-humic substances originate from viable cells and are relatively amenable to degradation, which generate altered molecular structure of new aquatic organic substances $^{49}$.Among these non-humified substances, carbohydrates are the most ubiquitous. These can be present as simple monosaccharide structures (composed of 3 to 6 carbon 
atoms) ${ }^{16}$ or more complexbranched polysaccharides (generally composed of 40 to 3000 carbon atoms) ${ }^{16}$. Among these carbohydrates, monosaccharides and disaccharides are the water soluble and biodegradable fractions ${ }^{50}$; whereas, starch and glycogen, the primary energy sources of plants and animals, respectively, are the most biodegradable fractions in polysaccharides $^{50}$. The availability of primary and secondary hydroxyl groups in starch and glycogen makes these polysaccharides hydrophilic in nature ${ }^{51}$. Other polysaccharides such as cellulose and chitins, on the other hand, are not readily biodegradable and neither are water soluble. These carbohydrates are comprised of uniform glucose structures which allow these to resist enzymatic breakdown ${ }^{52}$.

Proteins with amino acids as the primary building block(with amines and carboxyl functional moieties) are another important macromolecule in the aquatic environment, that can be structurally complex and can exhibit variation in surface charge (cationic, anionic, or non-ionic) ${ }^{53}$.Amino acids, such as cysteine and other proteins containing cys-residuals also possess thiol functional groups ${ }^{54}$, which enhance the affinity of these bio macro-molecules for some metal and metals oxides surface that are reactive to such disulfide and thiol groups ${ }^{55}$.Based on the environmental conditions, these thiol-containing proteins can form disulfide bonds, which might entirely change the interaction with NMs. Another prominent source of these non-humic substances is EPS, which can be excreted by both unicellular and multicellular organisms. The chemical composition of EPS varies depending on the organism from which these are produced ${ }^{56-58}$. EPS is typically responsible for conditioning environmental surfaces to allow formation of biofilms on granular media and sediments. Proteins and polysaccharides are the key components of EPS. Polysaccharides found in EPS can be either linear or branched and are primarily comprised of organic moieties such as Oacetyl, N-acetyl, succinyl, piruvyl as well as inorganic components such as sulfates and phosphates ${ }^{59}$. Other functional moieties such as amino, $\mathrm{CH}_{2}$, and $\mathrm{CH}_{3}$ groups are also be 
present in EPS ${ }^{59,60}$.The complexity in structure and variation in functional moieties of nonhumified carbohydrates and proteins calls for attention when assessing nano-EHS.

Humic substances on the other hand, are heavily decomposed and cannot be biodegraded by most aquatic organisms. While humic and fulvic acids are mostly water soluble, humins are insoluble in water at environmental $\mathrm{pHs}^{61,62}$. Humic substances can also demonstrate different elemental composition and physicochemical behavior depending on the aquatic environmental compartment of residence; i.e., ground water vs. surface water. Due to the presence of mineral collector surfaces in the subsurface, composition of NOM showsfunctional differences between the collector surfaces and porewater. Hydrophobic components of the NOM tend to adsorb onto mineral surfaces, leaving behind more hydrophilic components in the pore water as dissolved organic matter ${ }^{15}$. In comparison, surface water tends to possess a higher quantity of unbound and mobile humic fraction with a larger presence of hydrophobic chemical moieties compared to groundwater. The origin of these macromolecules is also largely different between these compartments due to the differences in the presence of biota and microorganisms. For instance, the fulvic acids in groundwater have relatively lower oxygen content that those in surface water. Thefulvic acids in the subsurface are also less aromatic in nature than those above surface because of a group of benzoate-metabolizing bacteria that resides in underground ${ }^{63}$. Such physicochemical and structural difference in organics and macromolecules will thus play a significant role in modifying NM interactionwith environmental interfaces ${ }^{64}$.

\section{NM Interaction with Macromolecules: Knowledge Gap and Challenges}

NMs can be released into the environment during their manufacturing process ${ }^{65,66}$, usage, and/orat the end-of-life disposal ${ }^{67-74}$, as well as can pass through wastewater facilities ${ }^{75,76}$. The EHS of these NMs thus is strongly affected by the environment these are released into or 
eventually might end up in.While studying the NM-macromolecule interaction, the general focus is on overall environmental conditions,i.e., typical composition of water in surface water or groundwater. These conditions can be considered relatively mild compared to extreme environments such as landfills, estuaries, and marshes. Non-humic and humic composition can vary widely in these environments, modulated by different organisms that reside in the respective areas. For example, landfills are repositories of abandoned products over a long period of time thus house a high concentration of organics compared to natural water and most soils ${ }^{77}$.Marshes have stagnant water also with high concentration of organic matter $^{78}$. Estuaries and coastal regions are highly saline and are perfectly conditioned for destabilizing colloidal particles and engineered NMs. It is extremely challenging to mimic such extreme conditions in the lab scale while studying the NM-macromolecule interaction. Hence, researchers have tried addressing such interaction with rather simple experimental designs. Several studies have highlighted the importance of NOM and complexity in NOM chemistry on aggregation, transport, and toxicological behavior of $\mathrm{NMs}{ }^{79-89}$. However, most of these studies use standardized humic and fulvic acids (e.g., Suwanee River humic acid and NOM) as representative NOMsnano-EHS studies ${ }^{79-85}$ and analyze the role NOM adsorption on nano-EHS. Though the complexity in NOM chemistry is appreciated in literature, little attention has been given to deconvolute the real-life complexity of NOMs.

Complexity of NM interaction in natural environment further increases from the presence of pre-existing surface coatings on engineered NMs, which are intentionally introduced to particle surfaces for achievinghigher colloidal stability ${ }^{90}$. Small charged molecules (e.g., citrate) and larger synthetic macromolecules, polymeric surfactants, polyelectrolytes (e.g., poly acrylic acid), or nonionic polymers (e.g., polyvinylpyrrolidone) are the most commonly used capping agents for engineered $\mathrm{NMs}^{91}$.Surfactants and polymers can be anionic, cationic, zwitterionic, and nonionic ${ }^{92}$, and can either be chemically bound via 
covalent bonding ${ }^{93,94}$, physisorbed by electrostatic or short ranged hydrophobic interactions ${ }^{95}$, ${ }^{96}$, or grafted ${ }^{97}$ ontoor from the NM surfaces. Different biomolecules are also introduced on NM surfaces as stabilizers, primarily in biomedical and therapeutic applications ${ }^{98}$; such biomolecules include: nucleic acids (i.e., DNA/RNA/PNA/LNA as genes, oligomers, aptamers, ribozymes/DNAzymes), antibody, fatty acids, lipids, mono and polysaccharides, different types of proteins, and peptides. ${ }^{99}$

The data gap in this literature further exacerbates via the expansion in the material space and development of complex nano-heterostructures ${ }^{100}$. These heterostructures can be classified as carbon-carbon, carbon-metal, core-shell meta-metal, among others ${ }^{16}$. Conjugation of multiple NMs result in the emergence of unique and synergistic electrical, optical, mechanical, catalytic, sensing ability and magnetic properties, and are being used for a wide range of applications is biomedical and nanotherapeutics, electro- and photocatalysis $^{101}$, electronics ${ }^{102}$, gas sensing ${ }^{103}$, biosensing ${ }^{104}$, laser technology ${ }^{105}$ and other emerging areas. Upon hybridization, these novel heterostructures have demonstrated emergent properties, which are different more than the sum of parts;e.g., hybridization of $\mathrm{TiO}_{2}$ with carbon nanotubes lowers band gap of the heterostructures and thus exhibits enhanced photocatalytic activity (visible photo-activation) and ROS generation compared to component $\mathrm{TiO}_{2}$ and $\mathrm{CNTs}^{106}$. The intrinsic van der Waals interaction energy of NMs are also found to be altered via hybridization ${ }^{107}$ and willlikely result in unknown interaction of the NHs with environmental interfaces (i.e., aggregation, deposition, and toxicity).

In order to address such wide data gap and reduce uncertainties in EHS studies, the field has utilized a wide range of state-of-the-art analytical techniques to characterize NM surfaces. In this quest, shift in NM surface Plasmon resonance due to the NOM, protein, and biopolymerbinding has been monitored ${ }^{4,}{ }^{108}$.Ellipsometry ${ }^{109}$, total reflection infrared spectroscopy $^{110,111}$, atomic force microscopy ${ }^{112}$ combined with flow field fractionation ${ }^{113,114}$, 
contact stylus instrument ${ }^{30}$, nuclear magnetic resonance with size-exclusion chromatography ${ }^{115}$, and other optical techniques such as dynamic light scattering ${ }^{116}$, circular dichroism ${ }^{117}$ have been applied to analyze adsorbed macromolecular layer thickness, ligand conformation, etc. Many of these techniques have effectively captured the protein/polymer layer thickness on the NM surfaces, however, have yet to successfully characterize ligand exchange and corona structure on NM surfaces. Ligand exchange is a dynamic process and depends on the thermodynamics of the system; thus, rate and extent of exchange need to be quantified. Moreover, NM-macromolecular corona conformation is also dynamic and is dependent on the chemistry of the macromolecule being exchanged as well as the surface chemistry of the NMs. A robust analytical technique is thus necessitated that can characterize tome-dependent macromolecular exchange as well as conformation of the corona to complete our understanding at the nano-bio interface.

\section{Probing the Bio-Accessibility of Nanoparticle-Ligand Conjugates}

\section{Single-Molecule Visualization of Polymeric Ligands on Nanomaterial Surfaces.We}

introduce a smTIRF-based microscopy platform ${ }^{118}$ to probe transient interactions of biomacromolecules on a NM surface at the single-molecule level. In this platform, fluorophorelabeled polymeric ligands serve to quantify and track in real-time the binding strength, binding kinetics, and propensity for ligand exchange of NM-adsorbing polymers on the surface of a single-walled carbon nanotube (SWNT) with unprecedented precision. We show that individual nanotube-bound polymeric ligands can be tracked in their interactions with a competing unlabeled ligand, resulting in partial desorption of the original ligand from the nanotube. Fluorescence microscopy is used to track the degree to which a competing ligand induces desorption of the original ligand, thus enabling a quantitative assessment of ligand binding to - and displacement of - a polymer-encapsulated nanoparticle. In our example, we use the known hybridization affinities of one DNA sequence with its complementary 
sequence to test the ability of smTIRF and visualize the hybridization process. In this manner, nanotube-bound polymeric ligandsare imaged via with smTIRF to observe the position and distribution of polymer ligandson the NM surface. In this platform, fluorescent polymeric ligands are adsorbed onto a SWNT surface, and the resulting polymer-SWNT moiety is surface-immobilized on a functionalized microscopy flow chamber. The polymer-SWNTs can then be studied in real-time upon exposure to different environmental conditions, controlled by fluid exchange in the $\sim 200 \mu \mathrm{L}$ microscopic imaging channel ${ }^{119}$.

Here, we present the visualization of three distinct polymer ligands adsorbed to the surface of SWNT: (i) Fifteen single-stranded DNA polynucleotide repeats of GT followed by a thirty-base random sequence((GT) $\left.)_{15}-\mathrm{Rnd}_{30}\right)$, (ii) fifteen consecutive GT repeats followed by a sixteen-base random sequence labeled with Cyanine 5 ((GT) $\left.)_{15}-\mathrm{Rnd}_{16-\mathrm{Cy}}\right)$, and (iii) Rhodamine B isothiocyanate (RITC) labeled polyethylene glycol (RITC-PEG-RITC). (GT) ${ }_{15^{-}}$ was chosen as a base sequence because each of the 30 aromatic bases of the $(\mathrm{GT})_{15}$ polymer provides a series of pi-stacking anchors for adsorption to the SWNTs, as we've shown previously ${ }^{120}$. Conversely, the RITC-PEG-RITC block copolymer only has two aromatic ends which can pi-stack to the SWNT, flanking a hydrophillic 5000 Da polyethylene glycol chain that does not interact with SWNT surface. Thus, the effects of a strongly-NM adsorbing $(G T)_{15}$ and a weakly-NM adsorbing RITC-PEG-RITC polymer can be independently studied. The Cy3 fluorophore, and RITC fluorophores, will quench when adsorbed to the SWNT due to fluorescence resonance energy transfer, and are expected to de-quench if desorbed from the SWNT.

Polymer-adsorbed nanotubes were preparedvia non-covalent adsorption of the polymeric ligand onto the nanotubes as described in Materials and Methods. Briefly, $0.2 \mathrm{mg}$ $(\mathrm{GT})_{15}-\mathrm{Rnd}_{30}$ or $(\mathrm{GT})_{15}$-Cy3 DNA polymers were mixed ina w/w 2:1 ratio with HiPCO SWNT in $1 \mathrm{~mL} 100 \mathrm{mMNaCl}$.The mixture was ultra-sonicated via tip sonication and 
subsequently purified via centrifugation to recover individually-dispersed (GT) ${ }_{15}$-Cy3 DNASWNT complexes (see supplemental Figure S2). The RITC-PEG-RITC polymer was adsorbed onto SWNTs, first by sonicating $0.2 \mathrm{mg}$ of HiPCO SWNT with a w/w excess of sodium dodecyl sulfate (SDS) and subsequently exchanging the SDS corona for RITC-PEGRITC at a concentration of $60 \mathrm{mg} / \mathrm{mL}$ with a $10 \mathrm{kDa}$ dialysis membrane in PBS buffer. Subsequent purification yielded individually-dispersed RITC-PEG-RITC-SWNT. Microfluidic imaging chambers for smTIRF microscopy were prepared using a BSA-biotin and NeutrAvidin surface immobilization protocol, as described previously ${ }^{121,122}$.

Imaging and quantifying DNA duplex formation on single SWNT. Non-fluorescent surfaceimmobilized (GT) $)_{15}-$ Rnd $_{30}$-SWNT were imaged via the addition of a short fluorescentlylabeled single-stranded DNA oligonucleotide complementary to the 30-base random sequencecarrying a 3'-terminally labeled Cy5 fluorophore tag Cy5-cRnd 30 . The appearance of fluorescence on the imaging surface thus corresponds to instances of DNA hybridization in which partial desorption of the $\mathrm{Rnd}_{30}$ polymer from the SWNT results from DNA hybridization by a complement strand. The test system of DNA strands, one originally bound to a SWNT, interacting via hybridization serves as a proxy for using smTIRF to study ligandnanoparticle interactions. Fig 2 shows the imaging surface upon addition ofCy5-cRnd 30 to surface-immobilized(GT) $)_{15}-\mathrm{Rnd}_{30}$-SWNT. Our imaging platform resolves Cy5-duplex labeled nanotubes, whereby the fluorescent signal originates from the in-situhybridization of Cy5-cDNA to the random 30 base ssDNA sequence on the SWNT ((GT) $)_{15}-\mathrm{Rnd}_{30}$-SWNT). We highlight that Cy5 cDNA hybridization results in a partial desorption of the ssDNA strand wrapping the SWNT with a hybridized duplex attached, and does not constitute a full desorption of the DNA from the SWNT.In Figure 2, the duplexes formed resulted in 103 fluorescent nanotubes within the sample field, in contrast to no fluorescence emission from the $(\mathrm{GT})_{15}-\mathrm{Rnd}_{30}$-SWNT only or Cy5-cRnd 30 only samples. We thus conclude that the 
association between the Cy5-labeled Cy5-cDNA oligonucleotide and the nanotube is due to DNA hybridization only, without non-specific adsorption of the oligonucleotide. The photobleaching of Cy5 fluorophores can be used to determine the number of unique duplexes per diffraction-limited DNA-SWNT spot. The intensity-time trace of a particular single nanotube shows discrete steps of comparable intensity corresponding to the photobleaching of 4Cy5molecules on the nanotube surface (Figure2d). Because each duplex contains a single fluorophore, the number of photobleaching steps observed per molecule represents the number of duplexes formed per functionalized nanotube. The number of duplexes per DNASWNT follows a non-normal skewed distribution with most SWNT spots showing 2 hybridized duplexes per SWNT, and anywhere between 1 and 4 duplexes observed per SWNT (Fig 2e, f). Adsorbing a low density of SWNT on the imaging surface ensures that most SWNT emitters within a $300 \mathrm{~nm}$ diffraction-limited spot will exist as singular SWNT per spot. However, we highlight that a small proportion of fluorescent spots could result from multiple SWNT within a diffraction-limited spot. As such, our platform enables direct optical tracking of ligands on nanomaterials. This imaging technique can thus be leveraged to study the adsorption of one ligand to another, demonstrated here by the hybridization of one DNA oligonucleotide to its SWNT surface-adsorbed complement.

\section{Quenching \& De-Quenching of Pi-Stacked Dyes as Proxy for Polymer}

\section{Conformation on SWNT Surface}

Imaging polymer desorption from a single SWNT via DNA hybridization.Quenching of organic fluorophores such as Cyanine 5 (Cy5), or RITC occurs as a function of proximity to the surface of SWNTs ${ }^{123}$, thereby creating a fluorescent ruler to quantify the degree of polymer desorption at a single-molecule scale. Quenching of fluorophores is expected for fluorophores adsorbed to the SWNT surface via pi-stacking, whereas brightening of 
fluorophores is expected from fluorophore de-quenching if the associated polymeric ligand partiallydesorbs from the surface. Surface-immobilized (GT) ${ }_{15}-$ Rnd $_{16}$-Cy5-SWNT were imaged prior to (Figure 3a) and after (Figure 3b) the addition of unlabeled cRnd ${ }_{16}$ oligonucleotide. (GT) ${ }_{15}-$ Rnd $_{16}$-Cy5-SWNT initially exhibit a large degree of Cy5 fluorophore quenching due to Cy5 pi-stacking to the SWNT surface. Addition of $\mathrm{cRnd}_{16^{-}}$ oligonucleotide promotes de-stacking of the Cy5 fluorophore via hybridization of the fluorophore-labeled $(\mathrm{GT})_{15}-\mathrm{Rnd}_{16}$-Cy5-SWNT, and results in de-quenching of the terminal Cy5. An average of $\mathrm{N}=50$ image captures shows the addition of $\mathrm{cRnd}_{16}$ increases the number of de-quenched Cy5 by $69.3 \%$, from $242 \pm 67$ Cy5 spots, to $410 \pm 68$ Cy5 spots (Figure 3c, error is standard error). As such, our platform allows quantification of the displacement of a polymeric ligand, $(\mathrm{GT})_{15}-\mathrm{Rnd}_{16}$, by a competing sequence-specific ligand, $\mathrm{cRnd}_{16}$, on the surface of a single-walled carbon nanotube.

Imaging polymer desorption from a single SWNT via ligand disruption.Here, we imaged surface-immobilized RITC-PEG-RITC SWNT and tested the change in the RITC-PEG-RITC ligand adsorption upon exposure to a competing ligand, estradiol.A $5 \mathrm{mg} / \mathrm{L}$ solution of RITCPEG-RITC SWNT were surface-immobilized in a microfluidic chamber with the same surface-adsorption scheme used for DNA-SWNTs (see Materials and Methods) within a $\sim 200 \mu \mathrm{L}$ volume, and initial RITC diffraction-limited fluorescent spots were counted.To quantify the conformation of the RITC-PEG-RITC polymer with respect to its SWNT surface-adsorption, counted the number of diffraction-limited SWNT molecules and RITC molecules in the absence or presence of $500 \mu \mathrm{M}$ estradiol. Upon addition of $500 \mu \mathrm{M}$ estradiol, we observe a $12 \%$ increase in the number of fluorescent RITC molecules per imaging field of view, from $271.7 \pm 4.5$ to $304.5 \pm 4.4$; mean \pm SE (Figure 4). We hypothesize that RITC fluorescence increase upon estradiol exposure is a result of the intercalation of estradiol between the aromatic carbons of the SWNT surface, and the RITC 
fluorophores of the RITC-PEG-RITC polymer. The physical intercalation of the estradiol analyte may yield separation between the SWNT surface and the RITC-PEG-RITC polymer without causing polymer desorption, thereby causing RITC de-quenching. A similar perturbation without full DNA polymer desorption was observed for DNA hybridization on SWNT in Figure 2 and 3.This direct relationship between a nanoparticle’s polymeric corona fluorescence and interaction with a competing (unlabeled) ligand may enable quantification of NOM adsorption to a polymer-functionalized nanoparticle. While this platform is presented as a modular interaction and partial displacement of a singular polymeric structure on a SWNT surface by a ligand, it can readily be extended to quantify multiple ligand-NOM interactions. Furthermore, smTIRF enables direct visualization and quantification of partially-desorbed phases, which is a missing dimension in the bulk corona ligand exchange described below.As such, we can use smTIRF microscopy to quantify the degree of polymer ligand disruption from its nanomaterial-adsorbed phase, when exposed to a competing ligand. Together, smTIRF with existing bulk-phase techniques can provide a full picture of partially and fully desorbed nanoparticle corona phases, and the extent to which these phases are perturbed by NOM ligands.

\section{Bulk Characterization of Nanoparticle Corona Ligand Exchange: UV-Vis-IR}

Spectroscopy. Orthogonal to smTIRF visualization of polymeric ligand corona exchange, UVVisible-Near Infrared absorption spectrometry, and near-infrared fluorescence emission spectrometry, can be utilized to validate the occurrence and degree to which a nanoparticle corona is exchanged for another ligand in bulk. Here, we compare the adsorption of either a RITC-PEG-RITC polymeric ligand, or sodium cholate (SC) surfactant, to SWNT. Using our RITC-PEG-RITC ligand adsorption to SWNT as a proxy, we demonstrate quantifiable tracking of RITC-PEG-RITC ligand exchange with a sodium cholate SWNT corona. Briefly, we first create a suspension of SC-adsorbed SWNT in water, using a $0.2 \mathrm{mg}$ mass of HiPCO 
SWNT and aw/w excess, 2\% solution of SC probe-tip sonicated for $1 \mathrm{~h}$ at $10 \mathrm{~W}$ power. Excess RITC-PEG-RITC is removed from solution through centrifugal filtration with a 100 $\mathrm{kDa}$ molecular weight cutoff prior to experimentation. The absorption (Figure5a) and fluorescence emission (Figure5b) spectra of $80 \mathrm{mg} /$ LRITC-PEG-RITC-SWNT show distinct peaks, each corresponding to a unique electronic transition within the SWNT, whose magnitude and frequency can be modulated by the dielectric SWNT environment. We show that the difference between a SC SWNT-adsorbed phase, and a RITC-PEG-RITC SWNTadsorbed phase, can be quantified by a solvatochromic shift in peak values.Normalized absorbance spectra of SWNTs in the near-infrared region before and after corona phaseexchange show that SWNT peaks corresponding to $E_{11}$ transitions(multiple chiralities between $900 \mathrm{~nm}$ and $1300 \mathrm{~nm}$ ) shift after replacement of SC by RITC-PEG-RITC on the SWNTsurface, while $E_{22}$ transitions (multiple chiralities between 600 - $900 \mathrm{~nm}$ ) remain comparable.Correspondingly, the near-infrared fluorescence of the two samples also reflect a change in relative peak intensities, as well as ared-shift in RITC-PEG-RITC-SWNT peak wavelength, corresponding to a morepolarizable environment provided by the PEG as compared to SDS.

\section{Materials and Methods}

\section{Materials}

Raw HipCO(High-pressure Carbon Monoxide synthesis process) SWNTs were purchased from NanoIntegris (batch \#HR27-104). All cyanine dye (Cy3 and Cy5) tagged ssDNA sequences were obtained from Integrated DNA Technologies (sequences below). Reagents were purchased from Sigma Aldrich unless otherwise specified. The following are polynucleotide sequences were used to encapsulate SWNTs, or to hybridize to DNAencapsulated SWNTs, as noted below: 
1) $\mathrm{DNA}=(\mathrm{GT})_{15}-\mathrm{Rnd}_{30}$, used to encapsulate SWNT:

2) $\quad$ cDNA $=$ Rnd $_{30}-\mathrm{Cy} 5$, hybridizes to DNA1 on SWNT:

3) DNA $=(G T)_{15}-$ Rnd $_{16}-$ Cy5-SWNT, used to encapsulate SWNT:

4) $\mathrm{cDNA}=\mathrm{cRnd}_{16}$, hybridizes to DNA1 on SWNT:

\section{DNA SWNT Suspension}

Solid SWNTs were added to a $100 \mathrm{mM} \mathrm{NaCl}$ solution in a 2:1 mass ratio with DNA. An Eppendorf tube containing $1 \mathrm{~mL}$ total volume of this mixture was placed in a cooling block and sonicated using an ultrasonic processor (Cole Parmer) with a $3 \mathrm{~mm}$ probe tip for $10 \mathrm{~min}$ at a power output of $6 \mathrm{~W}$. The resulting suspension was incubated at room temperature for 30 min then centrifuged at $16,000 \times$ g. $90 \%$ of the supernatant was collected so as to keep the pellet of SWNT aggregates intact. UV-vis absorbance (GE Nanovalue Plus, Fairfield, CT) was used to determine the concentration in mg/L of SWNT suspensions.

\section{smTIRF Microscopy of Immobilized SWNT Suspensions}

DNA SWNT suspensions were immobilized on the surface of a glass coverslip for fluorescence imaging. The surface of a glass microscope slide and \#1.5 glass coverslip was washed using isopropanol and water and then were dried. Strips of double-sided tape were laid across the microscope slide in parallel rows, $0.5 \mathrm{~cm}$ apart. The coverslip was then placed on top creating distinct microfluidic channels with volumes of approximately $20 \mu \mathrm{L}$ (see 
Figure S1).Each channel was first washed with $100 \mu \mathrm{L}$ of $100 \mathrm{mM} \mathrm{NaCl}$ solution by pipetting from one of the two openings and holding a Kimwipe on the other end to induce flow. Next, $50 \mu \mathrm{L}$ of biotinylated bovine serum Albumin in $100 \mathrm{mM} \mathrm{NaCl}$ (Thermo Scientific, $1 \mathrm{mg} / \mathrm{mL}$ ) was flowed through the channel. The BSA-biotin solution filled channel was incubated at room temperature for 5 min and then washed with $50 \mu \mathrm{L} \mathrm{NaCl}$ solution. The incubation and wash steps were repeated with NeutrAvidin $(0.2 \mathrm{mg} / \mathrm{mL}$, Thermo Scientific). Following rinsing of unbound NeutrAvidin, a $5 \mathrm{mg} / \mathrm{L}$ concentration of DNA-wrapped SWNTswere introduced into the imaging chamber, resulting in NeutrAvidin linked binding between DNASWNTs and the surface adsorbed BSA-biotin-NeutrAvidin, as described previously ${ }^{121,122}$. Immediately before imaging, all channels were flushed with $50 \mu \mathrm{L}$ of an oxygen scavenging buffer consisting of: $20 \mathrm{mM}$ Tris (pH 8), $200 \mathrm{mM} \mathrm{NaCl,} 2 \mathrm{mM}$ trolox, $0.16 \mathrm{mg} / \mathrm{mL}$ glucose oxidase, and $0.02 \mathrm{mg} / \mathrm{mL}$ catalase.

Microfluidic slides were imaged on a Zeiss Elyra microscope with a 100× TIRF objective. Cy3 and Cy5 were excited with laser of $561 \mathrm{~nm}$ and $642 \mathrm{~nm}$ wavelengths, respectively. A series of 1000 images were collected with 100 ms exposure. For samples which required imaging before and after hybridization, the complementary DNA was added in excess following the initial data collection. The channels were left to rest for 5 min then washed with imaging buffer a second time. The channels were then immediately imaged again using the same laser and detector settings.

\section{Conclusion}

Current experimental tools fall short to probe the structure of polymeric ligands on the surface of NMs during the dynamic changes in aqueous environments. Our work outlines the development of a fluorescence microscopy-based platform, which allows spatial and temporal resolution of polymeric structure and activity at this interface. Carbon nanotube- 
encapsulated nucleic acids and synthetic PEG-based block co-polymers, designed to explore polymer-dependent affinity to the nano-surface, are observed via total internal reflection (TIR) microscopy at the single-molecule level.Taking advantage of the single fluorophore resolution that allows us to observe individual ligand interactions with a SWNT, our measurements reveal novel dynamic properties and inter-molecular interactions of nano-scale systems that are not measurable from ensemble experiments. The application of single molecule fluorescence microscopy to NM provides evidence that ligand susceptibility to (i) targeted interaction with another ligand, (ii) intercalation or perturbation by another ligand, or (iii)full exchange by a competing ligand, is a process that can be imaged and quantified in real-time with smTIRF and infrared spectrometry.This platform, validated by several example applications, is generally applicable for nano-bio systems to answer questions of ligand structure, adsorption affinity, exchange propensity, and could enable future studies in environmental remediation and toxicity.This is another powerful toolset that will aid in resolving geo- and bio-macromolecular complexity at NM-water interfaces. Appreciating the complexity of chemical functionality and heterogeneity of natural organic matter, we note here that successful utilization of this technique for NOM binding will largely depend on deciphering specific spectral signature upon binding. Furthermore, single-molecule techniques are optimally suited to study single or few interactions. As such, single-molecule imaging will be most useful in its application to study the interaction of NOM and NOM surrogates with nanoparticles. We propose that analysis of NOM with the novel singlemolecule imaging technique ought to begin by utilizing NOM surrogate molecules(i.e., pyromellitic acid, salicylic acid, phthalic acid, thiol, and monosaccharide). Once relative nanomaterial-NOM surrogate binding or displacement strengths have been characterized, the platform can be extended to analyze complex geo- and bio-macromolecular corona characterization. 


\section{Acknowledgements}

This work was supported by a Burroughs Wellcome Fund Career Award at the Scientific Interface (CASI), the Simons Foundation, a BBRF young investigator award, and a Beckman Foundation Young Investigator Award (M.P.L.). It was also partially supported by National Science Foundation grant \#1440261 (N.B.S.).D.Y. acknowledges support from an NSF GRFP fellowship. Research reported in this publication was supported in part by the National Institutes of Health S10 program under award number 1S10OD018136-01. The content is solely the responsibility of the authors and does not necessarily represent the official views of the National Institutes of Health.

\section{References:}

1. G. V. Lowry, K. B. Gregory, S. C. Apte and J. R. Lead, Environmental Science \& Technology, 2012, 46, 6893-6899.

2. S. M. Louie, R. D. Tilton and G. V. Lowry, Environmental Science: Nano, 2016, 3, 283-310.

3. R. Amal, J. A. Raper and T. Waite, Journal of Colloid and Interface Science, 1992, 151, 244 257.

4. S. Diegoli, A. L. Manciulea, S. Begum, I. P. Jones, J. R. Lead and J. A. Preece, Science of the Total Environment, 2008, 402, 51-61.

5. A. A. Keller, H. Wang, D. Zhou, H. S. Lenihan, G. Cherr, B. J. Cardinale, R. Miller and Z. Ji, Environmental science \& technology, 2010, 44, 1962-1967.

6. D. Zhou and A. A. Keller, Water research, 2010, 44, 2948-2956.

7. Y. Zhang, Y. Chen, P. Westerhoff and J. Crittenden, Water Research, 2009, 43, 4249-4257.

8. K. Yang, D. Lin and B. Xing, Langmuir, 2009, 25, 3571-3576.

9. X. Wang, X. Chen, S. Liu and X. Ge, Journal of Environmental Sciences, 2010, 22, 1960-1965.

10. P. De Gennes, Advances in Colloid and Interface Science, 1987, 27, 189-209.

11. C. Tanford, The Hydrophobic Effect: Formation of Micelles and Biological Membranes $2 d$ Ed, J. Wiley., 1980.

12. M. A. Schlautman and J. J. Morgan, Geochimica et Cosmochimica Acta, 1994, 58, 4293-4303.

13. B. L. Lau, W. C. Hockaday, K. Ikuma, O. Furman and A. W. Decho, Colloids and Surfaces A: Physicochemical and Engineering Aspects, 2013, 435, 22-27. 
14. E. M. Murphy, J. M. Zachara and S. C. Smith, Environmental Science \& Technology, 1990, 24, 1507-1516.

15. R. Parfitt, A. Fraser and V. Farmer, Journal of Soil Science, 1977, 28, 289-296.

16. N. Aich, J. Plazas-Tuttle, J. R. Lead and N. B. Saleh, Environmental Chemistry, 2014, 11, 609623.

17. J. Plazas-Tuttle, L. Rowles, H. Chen, J. Bisesi, T. Sabo-Attwood and N. Saleh, Nanomaterials, 2015, 5, 1102.

18. N. B. Saleh, N. Aich, J. Plazas-Tuttle, J. R. Lead and G. V. Lowry, Environmental Science: Nano, 2015, 2, 11-18.

19. N. B. Saleh, A. Afrooz, J. H. Bisesi, N. Aich, J. Plazas-Tuttle and T. Sabo-Attwood, Nanomaterials, 2014, 4, 372-407.

20. I. A. Mudunkotuwa and V. H. Grassian, Environmental Science: Nano, 2015, 2, 429-439.

21. R. M. Jain, M. Ben-Naim, M. P. Landry and M. S. Strano, The Journal of Physical Chemistry C, 2015, 119, 22737-22745.

22. S. M. Louie, J. M. Gorham, E. A. McGivney, J. Liu, K. B. Gregory and V. A. Hackley, Environmental Science: Nano, 2016, 3, 1090-1102.

23. D.-H. Tsai, M. Davila-Morris, F. W. DelRio, S. Guha, M. R. Zachariah and V. A. Hackley, Langmuir, 2011, 27, 9302-9313.

24. M. C. Smith, R. M. Crist, J. D. Clogston and S. E. McNeil, Analytical and bioanalytical chemistry, 2015, 407, 3705-3716.

25. T. Cosgrove, T. G. Heath, K. Ryan and T. L. Crowley, Macromolecules, 1987, 20, 2879-2882.

26. M. Cárdenas, C. A. Dreiss, T. Nylander, C. P. Chan, T. Cosgrove and B. Lindman, Langmuir, 2005, 21, 3578-3583.

27. S. S. Karajanagi, A. A. Vertegel, R. S. Kane and J. S. Dordick, Langmuir, 2004, 20, 1159411599.

28. L. Treuel and G. U. Nienhaus, Biophysical Reviews, 2012, 4, 137-147.

29. T. Byrne, L. Lohstreter, M. Filiaggi, Z. Bai and J. Dahn, Surface Science, 2008, 602, 2927-2935.

30. R. Consiglio, N. X. Randall, B. Bellaton and J. von Stebut, Thin Solid Films, 1998, 332, 151-156.

31. T. Phenrat, N. Saleh, K. Sirk, H.-J. Kim, R. D. Tilton and G. V. Lowry, Journal of Nanoparticle Research, 2008, 10, 795-814.

32. K. E. Sapsford, K. M. Tyner, B. J. Dair, J. R. Deschamps and I. L. Medintz, Analytical Chemistry, 2011, 83, 4453-4488.

33. A. E. Nel, L. Mädler, D. Velegol, T. Xia, E. M. V. Hoek, P. Somasundaran, F. Klaessig, V. Castranova and M. Thompson, Nature Materials, 2009, 8, 543-557.

34. A. A. Shvedova, V. E. Kagan and B. Fadeel, Annual Reviews Pharmacology Toxicology, 2010, 50, 63-88.

35. T. S. Hauck, A. A. Ghazani and W. C. W. Chan, Small, 2008, 4, 153-159.

36. A. G. Beyene, G. S. Demirer and M. P. Landry, in Current Protocols in Chemical Biology, John Wiley \& Sons, Inc., 2009, DOI: 10.1002/cpch.10.

37. J. Zhang, M. P. Landry, P. W. Barone, J.-H. Kim, S. Lin, Z. W. Ulissi, D. Lin, B. Mu, A. A. Boghossian, A. J. Hilmer, A. Rwei, A. C. Hinckley, S. Kruss, M. A. Shandell, N. Nair, S. Blake, F. Sen, S. Sen, R. G. Croy, D. Li, K. Yum, J.-H. Ahn, H. Jin, D. A. Heller, J. M. Essigmann, D. Blankschtein and M. S. Strano, Nat Nano, 2013, 8, 959-968.

38. G. Bisker, J. Dong, H. D. Park, N. M. Iverson, J. Ahn, J. T. Nelson, M. P. Landry, S. Kruss and M. S. Strano, Nature Communications, 2016, 7, 10241.

39. S. Kruss, M. P. Landry, E. Vander Ende, B. M. A. Lima, N. F. Reuel, J. Zhang, J. Nelson, B. Mu, A. Hilmer and M. Strano, Journal of the American Chemical Society, 2014, 136, 713-724.

40. M. H. Wong, R. P. Misra, J. P. Giraldo, S.-Y. Kwak, Y. Son, M. P. Landry, J. W. Swan, D. Blankschtein and M. S. Strano, Nano Letters, 2016, 16, 1161-1172.

41. J. P. Giraldo, M. P. Landry, S.-Y. Kwak, R. M. Jain, M. H. Wong, N. M. Iverson, M. Ben-Naim and M. S. Strano, Small, 2015, 11, 3973-3984. 
42. J. P. Giraldo, M. P. Landry, S. M. Faltermeier, T. P. McNicholas, N. M. Iverson, A. A. Boghossian, N. F. Reuel, A. J. Hilmer, F. Sen, J. A. Brew and M. S. Strano, Nat Mater, 2014, 13, 400-408.

43. M. Landry, S. Kruss, J. Nelson, G. Bisker, N. Iverson, N. Reuel and M. Strano, Sensors, 2014, 14, 16196.

44. A. Jain, R. Liu, B. Ramani, E. Arauz, Y. Ishitsuka, K. Ragunathan, J. Park, J. Chen, Y. K. Xiang and T. Ha, Nature, 2011, 473, 484-488.

45. C. Niederer, R. P. Schwarzenbach and K.-U. Goss, Environmental science \& technology, 2007, 41, 6711-6717.

46. J. Mulder, M. Cresser, B. Moldan and J. Cerny, Biogeochemistry of small catchments, a tool for environmental research., 1994, 107-131.

47. R. L. Vannote, G. W. Minshall, K. W. Cummins, J. R. Sedell and C. E. Cushing, Canadian journal of fisheries and aquatic sciences, 1980, 37, 130-137.

48. J. Mao, W. Hu, K. Schmidt-Rohr, G. Davies, E. Ghabbour and B. Xing, Soil Science Society of America Journal, 2000, 64, 873-884.

49. S. Jose, Agroforestry systems, 2009, 76, 1-10.

50. W. Pigman, The carbohydrates: chemistry and biochemistry, Elsevier, 2012.

51. D. Lu, C. Xiao and S. Xu, Express polymer letters, 2009, 3, 366-375.

52. A. C. O'SULLIVAN, Cellulose, 1997, 4, 173-207.

53. N. M. Goldenberg and B. E. Steinberg, Cancer research, 2010, 70, 1277-1280.

54. M. V. Trivedi, J. S. Laurence and T. J. Siahaan, Current Protein and Peptide Science, 2009, 10, 614-625.

55. S. Aryal, B. Remant, N. Dharmaraj, N. Bhattarai, C. H. Kim and H. Y. Kim, Spectrochimica Acta Part A: Molecular and Biomolecular Spectroscopy, 2006, 63, 160-163.

56. R. S. Wotton, Scientia marina, 2004, 68, 13-21.

57. A. W. Decho, Oceanogr. Mar. Biol. Annu. Rev, 1990, 28, 73-153.

58. H.-C. Flemming and J. Wingender, Nature Reviews Microbiology, 2010, 8, 623-633.

59. J. Wingender, T. R. Neu and H.-C. Flemming, in Microbial extracellular polymeric substances, Springer, 1999, pp. 1-19.

60. Y.-P. Chen, P. Zhang, J.-S. Guo, F. Fang, X. Gao and C. Li, Chemosphere, 2013, 92, 633-638.

61. E. M. Thurman and R. L. Malcolm, Environmental Science \& Technology, 1981, 15, 463-466.

62. M. Schnitzer, Methods of Soil Analysis. Part 2. Chemical and Microbiological Properties, 1982, 581-594.

63. C. Pettersson, J. Ephraim and B. Allard, Organic Geochemistry, 1994, 21, 443-451.

64. E. M. Murphy and J. M. Zachara, Geoderma, 1995, 67, 103-124.

65. D. Bello, A. J. Hart, K. Ahn, M. Hallock, N. Yamamoto, E. J. Garcia, M. J. Ellenbecker and B. L. Wardle, Carbon, 2008, 46, 974-977.

66. B. Yeganeh, C. M. Kull, M. S. Hull and L. C. Marr, Environmental Science \& Technology, 2008, 42, 4600-4606.

67. J. Farkas, H. Peter, P. Christian, J. A. Gallego Urrea, M. Hassellöv, J. Tuoriniemi, S. Gustafsson, E. Olsson, K. Hylland and K. V. Thomas, Environment International, 2011, 37, 1057-1062.

68. L. Geranio, M. Heuberger and B. Nowack, Environmental Science \& Technology, 2009, 43, 8113-8118.

69. R. Kaegi, B. Sinnet, S. Zuleeg, H. Hagendorfer, E. Mueller, R. Vonbank, M. Boller and M. Burkhardt, Environmental Pollution, 2010, 158, 2900-2905.

70. R. Kaegi, A. Ulrich, B. Sinnet, R. Vonbank, A. Wichser, S. Zuleeg, H. Simmler, S. Brunner, H. Vonmont, M. Burkhardt and M. Boller, Environmental Pollution, 2008, 156, 233-239.

71. T. M. Benn and P. Westerhoff, Environmental Science \& Technology, 2008, 42, 4133-4139.

72. T. Benn, B. Cavanagh, K. Hristovski, J. D. Posner and P. Westerhoff, J. Environ. Qual., 2010, $39,1875-1882$. 
73. L.-Y. Hsu and H.-M. Chein, in Nanotechnology and Occupational Health, eds. A. Maynard and D. H. Pui, Springer Netherlands, 2007, DOI: 10.1007/978-1-4020-5859-2_15, ch. 15, pp. 157163.

74. A. R. Köhler, C. Som, A. Helland and F. Gottschalk, Journal of Cleaner Production, 2008, 16, 927-937.

75. M. A. Kiser, P. Westerhoff, T. Benn, Y. Wang, J. Pérez-Rivera and K. Hristovski, Environmental Science \& Technology, 2009, 43, 6757-6763.

76. R. Kaegi, A. Voegelin, B. Sinnet, S. Zuleeg, H. Hagendorfer, M. Burkhardt and H. Siegrist, Environmental Science \& Technology, 2011, 45, 3902-3908.

77. D. Kulikowska and E. Klimiuk, Bioresource Technology, 2008, 99, 5981-5985.

78. C. C. Tanner, J. P. Sukias and M. P. Upsdell, Water Research, 1998, 32, 3046-3054.

79. A. R. M. N. Afrooz, I. A. Khan, S. M. Hussain and N. B. Saleh, Environmental Science \& Technology, 2013, 47, 1853-1860.

80. A. R. M. N. Afrooz, S. T. Sivalapalan, C. J. Murphy, S. M. Hussain, J. J. Schlager and N. B. Saleh, Chemosphere, 2013, 91, 93-98.

81. N. Aich, L. K. Boateng, I. V. Sabaraya, D. Das, J. R. Flora and N. B. Saleh, Environmental science \& technology, 2016, 50, 3562-3571.

82. I. A. Khan, A. R. M. N. Afrooz, J. R. V. Flora, P. A. Schierz, P. L. Ferguson, T. Sabo-Attwood and N. B. Saleh, Environmental Science \& Technology, 2013, 47, 1844-1852.

83. I. A. Khan, J. R. V. Flora, A. R. M. N. Afrooz, N. Aich, P. A. Schierz, P. L. Ferguson, T. SaboAttwood and N. B. Saleh, Environmental Chemistry, 2015, 12, 652-661.

84. N. B. Saleh, L. D. Pfefferle and M. Elimelech, Environmental Science \& Technology, 2008, 42, 7963-7969.

85. A. R. M. N. Afrooz, D. Das, C. J. Murphy, P. Vikesland and N. B. Saleh, Water Research, 2016, 99, 7-15.

86. D. Lin, X. Tian, F. Wu and B. Xing, Journal of Environmental Quality, 2010, 39, 1896-1908.

87. S. Kang, M. S. Mauter and M. Elimelech, Environmental science \& technology, 2009, 43, 2648-2653.

88. M. Baalousha, A. Manciulea, S. Cumberland, K. Kendall and J. R. Lead, Environmental Toxicology and Chemistry, 2008, 27, 1875-1882.

89. E. M. Hotze, T. Phenrat and G. V. Lowry, Journal of environmental quality, 2010, 39, 19091924.

90. J. Buffle, K. J. Wilkinson, S. Stoll, M. Filella and J. Zhang, Environmental Science \& Technology, 1998, 32, 2887-2899.

91. C. N. R. Rao, A. Müller and A. K. Cheetham, The chemistry of nanomaterials: synthesis, properties and applications, John Wiley \& Sons, 2006.

92. P. Somasundaran and S. Krishnakumar, Colloids and Surfaces A: physicochemical and engineering aspects, 1997, 123, 491-513.

93. H. Zeng, C. Gao, Y. Wang, P. C. Watts, H. Kong, X. Cui and D. Yan, Polymer, 2006, 47, 113-122.

94. G. Chao, L. Wenwen, J. Yi Zheng and K. Hao, Nanotechnology, 2006, 17, 2882.

95. I. A. Khan, J. R. Flora, A. N. Afrooz, N. Aich, P. A. Schierz, P. L. Ferguson, T. Sabo-Attwood and N. B. Saleh, Environmental Chemistry, 2015, 12, 652-661.

96. N. Aich, L. K. Boateng, J. R. Flora and N. B. Saleh, Nanotechnology, 2013, 24, 395602.

97. S. K. Kumar, N. Jouault, B. Benicewicz and T. Neely, Macromolecules, 2013, 46, 3199-3214.

98. X. Wang, L.-H. Liu, O. Ramström and M. Yan, Experimental biology and medicine (Maywood, N.J.), 2009, 234, 1128-1139.

99. K. E. Sapsford, W. R. Algar, L. Berti, K. B. Gemmill, B. J. Casey, E. Oh, M. H. Stewart and I. L. Medintz, Chemical Reviews, 2013, 113, 1904-2074.

100. W. A. Rigdon and X. Huang, Journal of Power Sources, 2014, 272, 845-859.

101. D. Eder and A. H. Windle, Advanced Materials, 2008, 20, 1787-1793.

102. N. J. Alley, K. S. Liao, E. Andreoli, S. Dias, E. P. Dillon, A. W. Orbaek, A. R. Barron, H. J. Byrne and S. A. Curran, Synth. Met., 2012, 162, 95-101. 
103. E. Llobet, E. Espinosa, E. Sotter, R. lonescu, X. Vilanova, J. Torres, A. Felten, J.-J. Pireaux, X. Ke and G. Van Tendeloo, Nanotechnology, 2008, 19, 375501.

104. Z. Liu, J. Wang, D. Xie and G. Chen, Small, 2008, 4, 462-466.

105. Y. Zhu, H. I. Elim, Y. L. Foo, T. Yu, Y. Liu, W. Ji, J. Y. Lee, Z. Shen, A. T.-S. Wee and J. T.-L. Thong, Advanced Materials, 2006, 18, 587-592.

106. K.-h. Jung, J. S. Hong, R. Vittal and K.-J. Kim, Chemistry Letters, 2002, 864-865.

107. Z. Hua, J. Zhang, X. Bai, Z. Ye, Z. Tang, L. Liang and Y. Liu, Science of The Total Environment, 2016, 539, 196-205.

108. M. Lundqvist, I. Sethson and B.-H. Jonsson, Langmuir, 2004, 20, 10639-10647.

109. S. H. Brewer, W. R. Glomm, M. C. Johnson, M. K. Knag and S. Franzen, Langmuir, 2005, 21, 9303-9307.

110. P. Yang, X. F. Meng, Z. Y. Zhang, B. X. Jing, J. Yuan and W. T. Yang, Analytical Chemistry, 2005, 77, 1068-1074.

111. A. Vargas, I. Shnitko, A. Teleki, S. Weyeneth, S. E. Pratsinis and A. Baiker, Appl. Surf. Sci., 2011, 257, 2861-2869.

112. D. K. Kim, M. Mikhaylova, Y. Zhang and M. Muhammed, Chem. Mat., 2003, 15, 1617-1627.

113. M. Baalousha and J. R. Lead, Environmental Science \& Technology, 2007, 41, 1111-1117.

114. M. Baalousha and J. R. Lead, Science of the Total Environment, 2007, 386, 93-102.

115. E. Hellstrand, I. Lynch, A. Andersson, T. Drakenberg, B. Dahlbäck, K. A. Dawson, S. Linse and

T. Cedervall, Febs Journal, 2009, 276, 3372-3381.

116. N. Inomoto, N. Osaka, T. Suzuki, U. Hasegawa, Y. Ozawa, H. Endo, K. Akiyoshi and M.

Shibayama, Polymer, 2009, 50, 541-546.

117. C.-C. You, M. De, G. Han and V. M. Rotello, Journal of the American Chemical Society, 2005, 127, 12873-12881.

118. R. Roy, S. Hohng and T. Ha, Nature Methods, 2008, 5, 507-516.

119. P. V. Jena.

120. M. P. Landry, L. Vuković, S. Kruss, G. Bisker, A. M. Landry, S. Islam, R. Jain, K. Schulten and M. S. Strano, The Journal of Physical Chemistry C, 2015, 119, 10048-10058.

121. L. Chio, D. Yang and M. Landry, in Synthetic Antibodies: Methods and Protocols, ed. T. Tiller, Springer New York, New York, NY, 2017, DOI: 10.1007/978-1-4939-6857-2_23, pp. 363-380.

122. M. P. Landry, H. Ando, A. Y. Chen, J. Cao, V. I. Kottadiel, L. Chio, D. Yang, J. Dong, T. K. Lu and M. S. Strano, Nat Nano, 2017, advance online publication.

123. R. Yang, J. Jin, Y. Chen, N. Shao, H. Kang, Z. Xiao, Z. Tang, Y. Wu, Z. Zhu and W. Tan, J Am Chem Soc, 2008, 130, 8351-8358. 


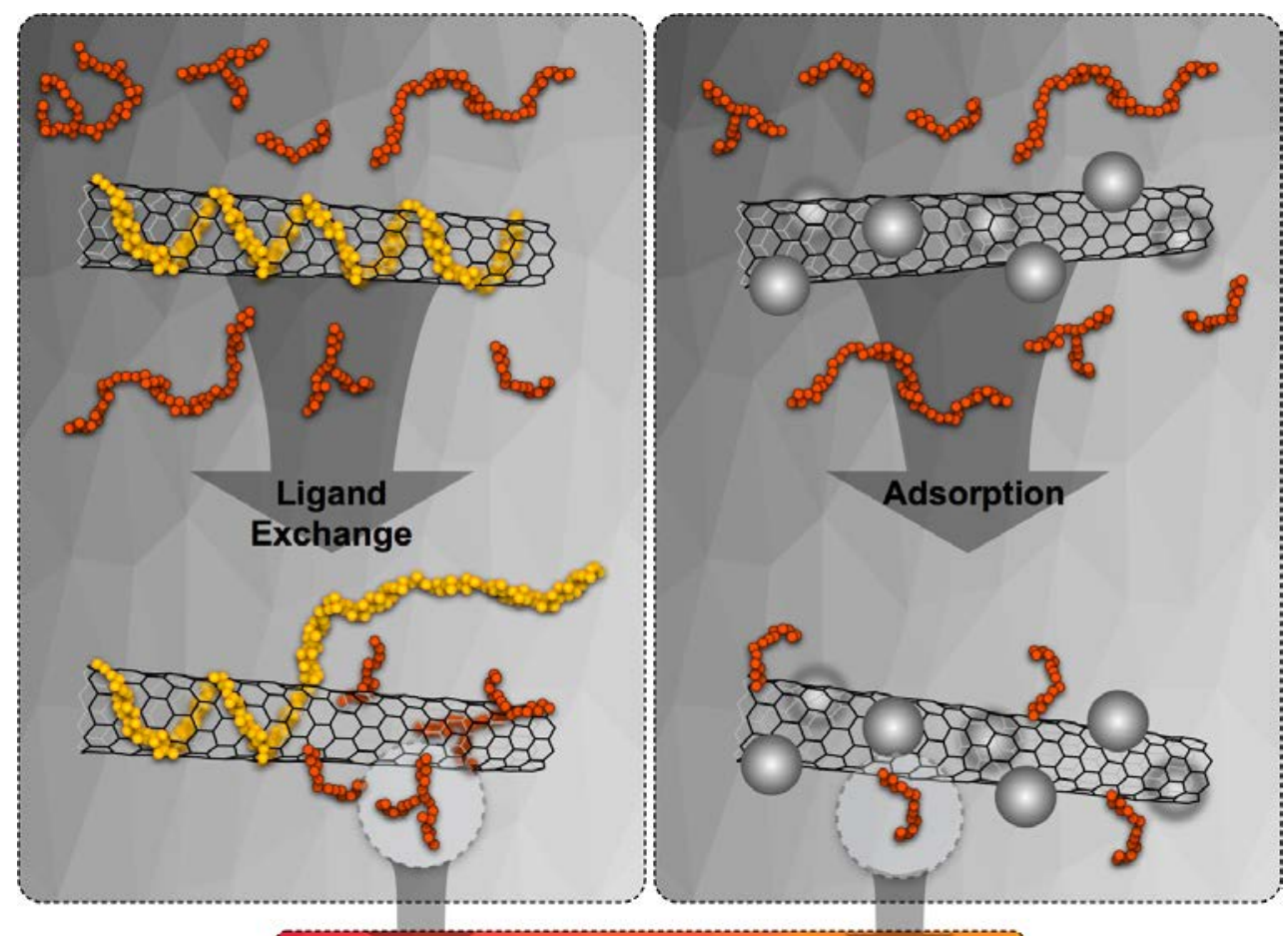

\section{SINGLE MOLECULE IMAGING}

Figure 1. Schematic representing macromolecular adsorption and ligand exchange on NM surfaces, which can be systematically characterized with single molecule imaging. 


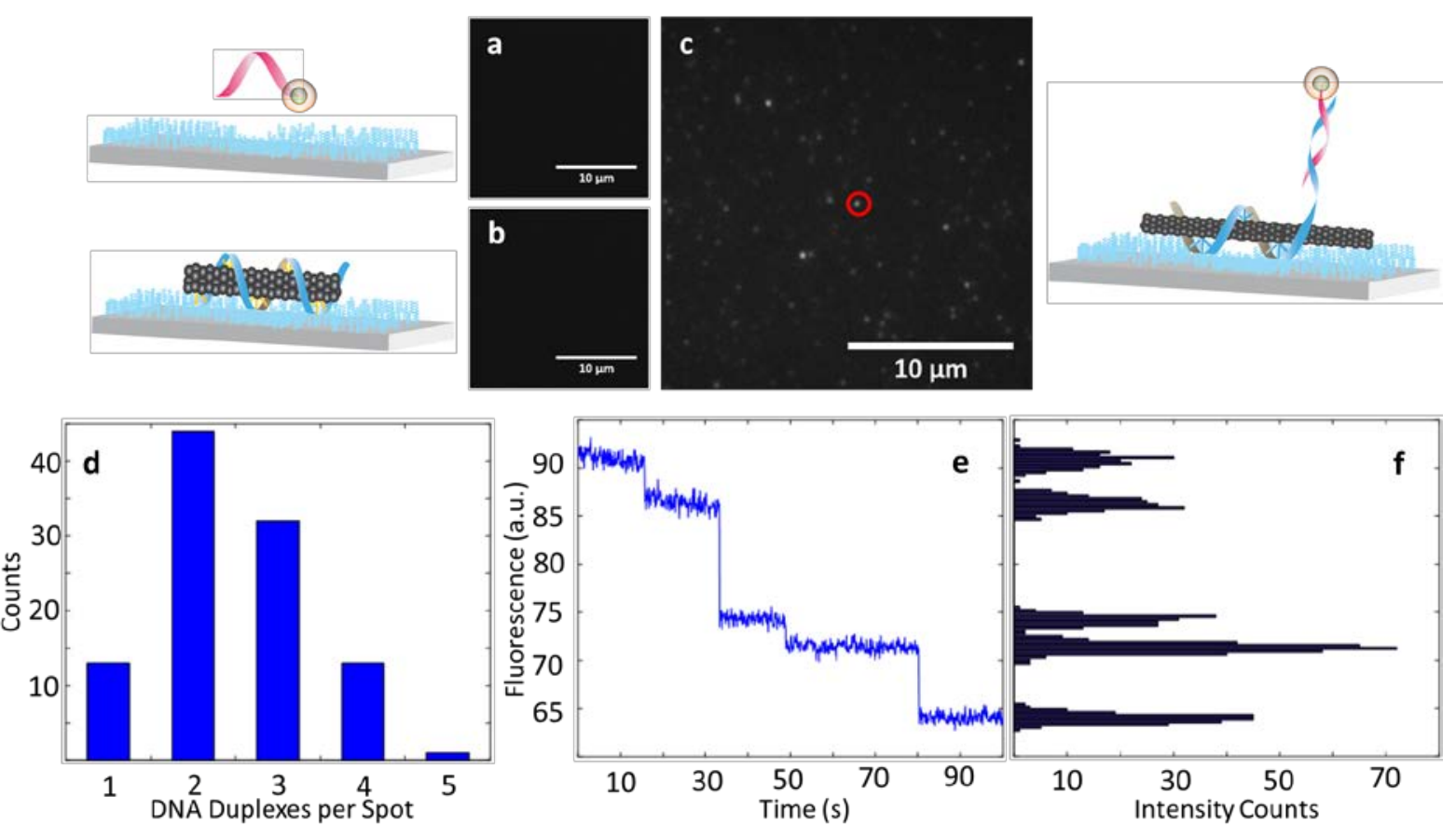

Figure 2.(a) Cy5-cRnd 30 only and (b) $(\mathrm{GT})_{15}-\mathrm{Rnd}_{30}-\mathrm{SWNT}$ only, on the imaging surface, show no fluorescence (c) Fluorescence is due to Cy5 specifically, not due to non-specific adsorption of contaminants or stray Cy5-cRnd 30 ( $n>20$ for all controls) (d) counts per SWNT spot of Cy5 intensity quenching enable quantification of DNA duplexes formed per SWNT spot. Corresponding (e) Intensity time trace (100 ms time resolution) of a Cy5-cDNA hybridized to an immobilized (GT) $)_{15}$-Rnd ${ }_{30}$-SWNT complex shows 4 photobleaching steps and corresponding (f) fluorescence intensity counts, each representing to a unique Cy5-cDNA ligand. 

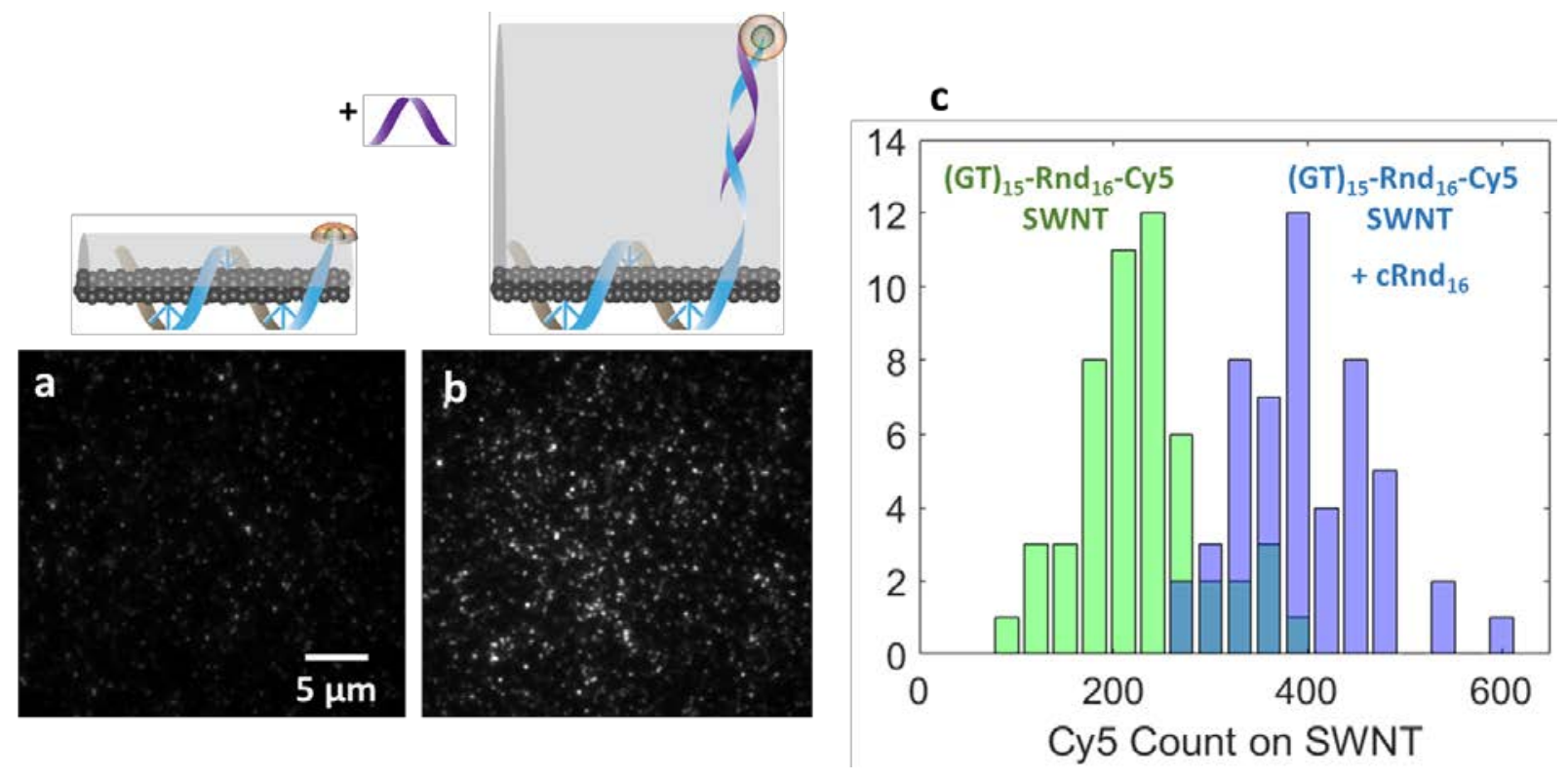

Figure 3.smTIRF fluorescence microscopy of DNA-carbon nanotubes.(a) Nanotubes encapsulated with $(\mathrm{GT})_{15}-\mathrm{Rnd}_{16}$-Cy5 polymer ligand show quenched Cy5 fluorescence (b) addition of $\mathrm{CRND}_{16}$ hybridizes to the $(\mathrm{GT})_{15}-\mathrm{Rnd}_{16}-\mathrm{Cy} 5$ and results in Cy5 de-stacking from the SWNT, and de-quenching in the fluorescence microscope (scale bar $5 \mu \mathrm{m}$ ). Quantification of all fluorescent ligands on all nanotubes in the sample shows an increase in de-quenched fluorescent SWNT spots after addition of unlabeled cRnd ${ }_{16}$. 

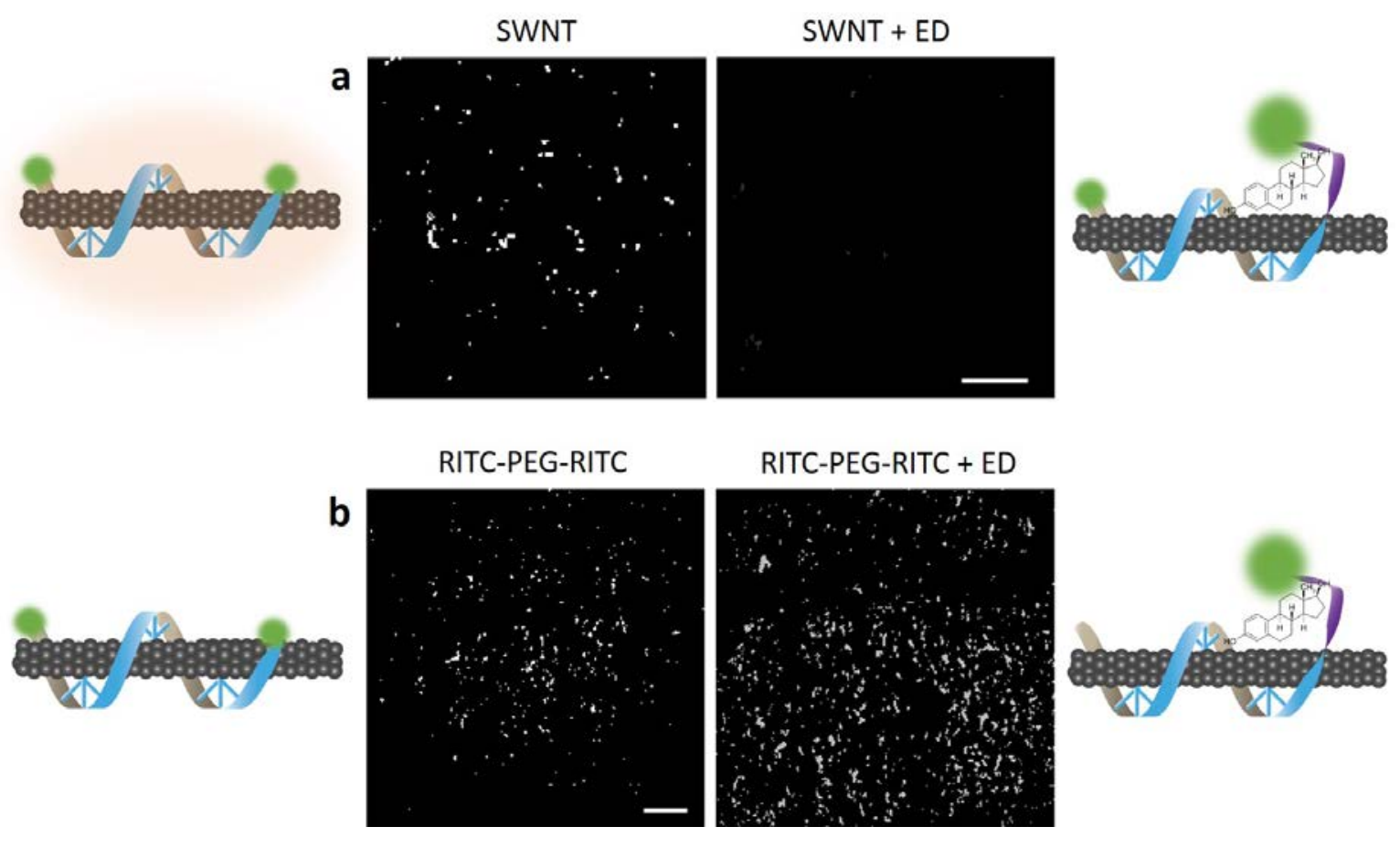

Figure 4. Effect of estradiol on RITC-PEG-RITC: infrared SWNT fluorescence and visible RITC fluorescence. Upon addition of $500 \mu \mathrm{M}$ estradiol to individually surfaceimmobilized RITC-PEG-RITC-SWNT (a) Near-infrared fluorescence of SWNT quenches with after exposure to estradiol. (b) Visible fluorescence of the RITC fluorophore dequenches after exposure to estradiol. Scale $1 \mu \mathrm{m}$. 

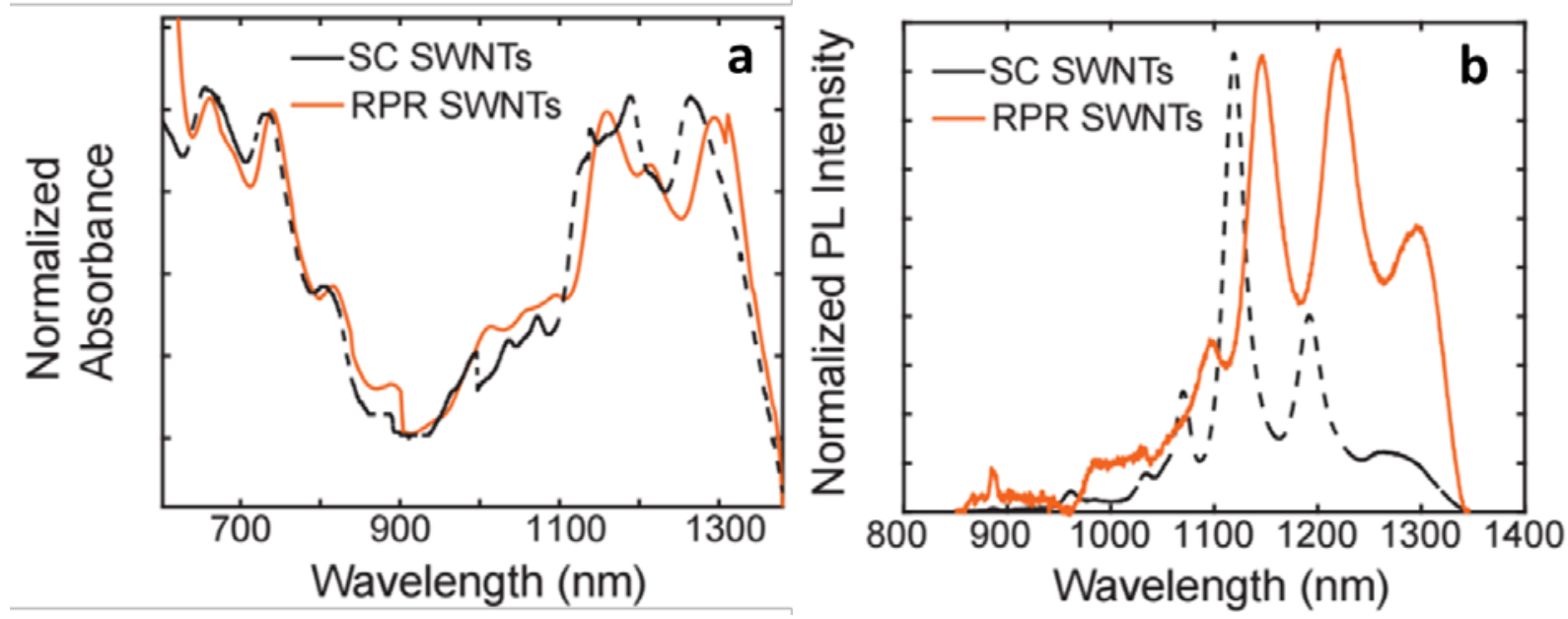

Figure 5.UV-Vis-IR Absorption, and near-IR fluorescence spectroscopy characterization of RITC-PEG-RITC polymer ligands.(a)Absorption spectraof SWNT are red-shifted when RITC-PEG-RITC is adsorbed on the SWNT (orange), in comparison to sodium cholate adsorbed to SWNT (black, dashed). (b)Corresponding fluorescence emission spectra with $721 \mathrm{~nm}$ excitationof RITC-PEG-RITC adsorbed SWNT (orange) red-shifted in comparison to sodium cholate adsorbed to SWNT (black).Time between spectra is $1 \mathrm{~h}$. 


\section{Supplemental Information}

\section{Importance and challenges of environmental ligand binding and exchange: Introducing single molecule imaging as a model characterization technique}

By

${ }^{1}$ Navid B. Saleh, ${ }^{1}$ Dipesh Das, ${ }^{1}$ Jaime Plazas-Tuttle, ${ }^{2}$ Darwin Yang, ${ }^{2}$ Jackson Travis DelBonisO'Donnell, and ${ }^{2,3}$ Markita P. Landry ${ }^{*}$

${ }^{1}$ Department of Civil, Architectural and Environmental Engineering, University of Texas at Austin, Austin, TX 78712

${ }^{2}$ Department of Chemical and Biomolecular Engineering, University of California-Berkeley, Berkeley, CA 94720

${ }^{3}$ California Institute for Quantitative Biosciences (qb3), University of California-Berkeley, Berkeley, CA 94720

In preparation for Submission

to

ES: Nano (Expected: November, 2016)

*Corresponding author: Markita P. Landry, email: landry@berkeley.edu, phone: (510) 6647627 